\title{
Surface models of granular materials in storage tanks
}

\author{
Nikolaj Zamyatin ${ }^{1, *}$, Vladimir Makovkin ${ }^{1}$, and Gennadij Smirnov ${ }^{1}$ \\ ${ }^{1}$ Tomsk State University of Control Systems and Radioelectronics, 634045 Tomsk, Russia
}

\begin{abstract}
The paper focuses on the problem of measuring the volume of granular materials in storage tanks where, due to uneven surfaces of these materials, the error in measurement by conventional methods reaches $15-$ 20 percent. To increase the accuracy of volume measurement, a technique for modeling surfaces formed by granular materials in tanks is proposed.
\end{abstract}

\section{Introduction}

Enterprises of the construction industry, chemical plants, agricultural companies and enterprises of other industries use a variety of tanks (tank batteries) to store raw materials, finished products, various granular substances and materials, and should operate these tanks as efficiently as possible [1-4].

If a material is granular, viscous or has a consistency other than liquid, it forms an uneven surface of convex or concave shapes (depending on whether materials are supplied to or pumped from a tank). A curved surface of materials is formed by cohesive forces that make particles coalesce with each other and with walls of the tank (Fig. 1). The most common method of volume measurement in tanks is to measure the surface level at only one point and then average the data, but this method should generally be used for liquid materials $[5,6]$. Such a volume measurement method leads to low accuracy of data for tank batteries and, therefore, has a direct influence on economic losses of enterprises. This results in downtime due to miscalculated dynamics of consumption or production of raw materials; low quality of mixtures due to errors in calculating ratios of ingredients in different formulations; additional costs arising from related accounting errors; miscalculated production volumes and consumption of raw materials, and other problems.

\footnotetext{
*Corresponding author: zamnv47@gmail.com
} 


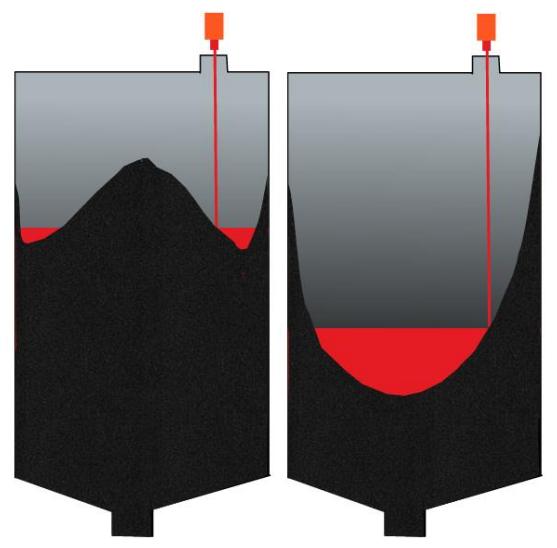

Fig. 1. Shape of the surface when supplying the material to/pumping it from the tank.

In order to increase the accuracy in practice, researchers model the surface of materials using known coordinates and, for this purpose, rely on mathematical surface relief models, in particular by approximating heights between adjacent points of the surface model [7]. More general statistical models include the "floating square" and "floating circle" described in [8].

Volume calculation is based on different techniques and depends on required accuracy [9]. This study uses a laser method to measure the surface level and three modeling techniques: by smooth surface, by cone, and by uneven surface, with neural network approximation and volume calculation through numerical integration. Similar studies are performed in Russia and abroad for other level measurement tools [5, 6, 9].

\section{Materials and Methods}

According to the proposed technique, volume measurement for granular materials in a storage tank is divided into the following stages:

1) determining a discrete set of values of surface levels;

2) approximating the surface to determine intermediate unknown values of coordinates and its nonlinear function;

3) calculating the volume of material under the formed surface.

The volume measurement method by smooth surface lies in measuring the level at a certain location and calculating the volume of material. This is the simplest method that is usually applied to control the volume of liquid materials in case of manual measurement. It is the fastest method among the considered ones and does not require complex calculation. However, since this method ignores rheological and flow properties of mixtures, it has the lowest accuracy for granular materials as compared to all other methods proposed above.

The volume measurement method by cone lies in measuring the level of material at a specific location and modeling the cone or conical depression (for cases when the material is supplied to and pumped from the tank, respectively). The angle between generatrices and base is equal to the angle of repose of the material. The angle of repose (sometimes the angle of internal friction or angle of slope) is the angle formed by a free surface of loose mass or other granular material with a horizontal plane.

By using the angle of repose and calculating the cone volume, it is possible to significantly increase the accuracy as compared to volume measurement by smooth surface. However, this method has the following disadvantages:

- it requires more data and preliminary calculations for different types of tanks; 
- the angle of repose varies depending on moisture, type and grade of granular materials and is determined experimentally;

- the angle between the base and loose mass is equal to the angle of repose only when supplying to or pumping materials from the tank. However, when switching from the first process to the second one and vice versa, the angle will change too.

The volume under an uneven surface in a vertical cylindrical tank is determined subject to the geometric meaning of the double integral. If the $f(x, y)$ function takes only positive values in the $D$ domain, the double integral of this function is numerically equal to the volume $V$ of the vertical cylindrical body constructed on the basis of $D$ and bounded above by the corresponding part of the surface $z=f(x, y)[10]$.

$$
V=\iint_{D} z d x d y
$$

Values of integrals can be calculated in two common ways: either using quadrature or cubature formulas, or using the Monte Carlo method.

For mathematical modeling of surfaces, it is suggested to use neural networks that have high speed and accuracy of models. In [7, 11], various neural networks were compared, in particular for approximation of input values. In [11], the output values of neural networks were compared according to the coefficients of determination presented in Table 1.

Table 1. Quality of approximation based on the coefficients of determination.

\begin{tabular}{|c|c|c|c|}
\hline & $\begin{array}{c}\text { GRNN } \\
\text { network }\end{array}$ & $\begin{array}{c}\text { RBF } \\
\text { network }\end{array}$ & $\begin{array}{c}\text { Linear } \\
\text { network }\end{array}$ \\
\hline Coefficient $\left(R^{2}\right)$ & 0.9473 & 0.9918 & 0.6422 \\
\hline
\end{tabular}

When smoothing the function on the basis of models other than neural networks, such as Fourier series and a multiplicative model, the coefficients of determination are equal to 0.8747 and 0.7262 , respectively.

From the data shown above, it is clear that RBF and GRNN neural networks are best suited for approximating surfaces. The RBF network gives the coefficient of determination $R^{2}=0.9918$. This means that the actual output of the neural network and the desired output are almost identical. Using analytical or numerical methods, it is practically impossible to achieve such a high value of the coefficient of determination. In terms of processing speed and network learning, RBF networks are best suited for approximating the surface model, since they do not have the second intermediate layer that would be responsible for estimating the weighted mean, as in case of GRNN. Therefore, RBF networks can be used to model the surface of materials.

In 1956, Kolmogorov proved that any continuous function can be implemented as a superposition of functions of smaller dimension [12]. The implicit function of threedimensional surface can be represented in the form (2):

$$
f(x, y)=\sum_{i=1}^{n} \omega_{i} \varphi(r),
$$

where $\omega_{i}$ are the constants; $\varphi(r)$ is the radial basis function.

As a function of $\varphi(r)$, we can choose any radial basis function (3) that is used to solve problems of multidimensional interpolation and approximation.

$$
\varphi\left(x, y, c_{i}^{x}, c_{i}^{y}\right)=1-\sqrt{\left(x-c_{i}^{x}\right)^{2}+\left(y-c_{i}^{y}\right)^{2}}
$$


where $c^{x}{ }_{i}, c^{y}{ }_{i}$ are some constants, the so called RBF kernels.

The implicit surface function can be implemented using a neural network consisting of two layers: a hidden nonlinear layer with $\mathrm{n}$ neurons and an output linear layer with one neuron that transmits a weighted sum of neuron outputs of the first layer to the output.

The RBF neural network learning algorithm is as follows:

- to select the number of neurons in the hidden layer so that it is equal to the number of input points;

- to specify a RBF kernel for each neuron $\left(c^{x}, c^{y}{ }_{i}\right)$ so that $c^{x}{ }_{i}=x_{i}, c^{y}{ }_{i}=y_{i}$;

- to determine weights $\omega_{i}$.

The output value of the network for the ith input data is determined by the formula (4)

$$
D_{i}=w_{1} f\left(x_{i}, y_{i}, c_{1}^{x}, c_{1}^{y}\right)+w_{2} f\left(x_{i}, y_{i}, c_{2}^{x}, c_{2}^{y}\right)+\ldots+w_{n} f\left(x_{i}, y_{i}, c_{n}^{x}, c_{n}^{y}\right)
$$

Thus, the surface relief is represented in the form of a nonlinear function - a superposition of the learned RBF neural network. Then the volume of a certain material can be determined by solving a double or triple integral of an already known function.

The error in calculating the additional volume depends only on surface irregularities, since the volume of material below the minimum surface level of the mixture is determined by the shape and dimensions of the tank. The volume of material can be measured by the following methods.

\section{Results}

The possibility of using the proposed method was tested experimentally based on a model of vertical cylindrical tank in which M400 cement was used as a material to be measured. To measure the level, we used a cylindrical coordinate system with the $\mathbf{0 z}$ axis passing through the center of the model. In real conditions, non-uniformly distributed data can be transmitted to the input of a RBF neural network. However, for the sake of simplicity, the points were equally spaced from each other, while level values were measured at equal intervals $\varphi$ and $r$ from each other.

A SICKDT50 (error $\pm 1 \mathrm{~mm}$ ) laser rangefinder was used as a level meter.

Since the applied radial basis function does not work with cylindrical coordinates directly because of the Euclidean distance between the center and the points, it is necessary to change variables through a Jacobian.

A cubic RBF function is used as an activation neuron function. The activation function was chosen experimentally while comparing with the real surface by the appearance of the model:

$$
a=n^{3}
$$

When teaching the RBF neural network with zero error, the number of neurons in the hidden layer is always equal to the number of elements in the learning set (values transmitted to the network input). With such an error, it is impossible to obtain an acceptable (in terms of speed) solution for large training sets. Therefore, additional manipulations are required to minimize the error of the model. Moreover, the zero error does not guarantee that the intermediate values for which the neural network has not been learned will give an accurate result. On the contrary, the surface model will be more likely to have a complex shape that is different from the real one.

As shown by experiments, twelve neurons were sufficient to learn a neural network with an error of less than one percent, while significantly reducing the calculation time. The performance of the neural network with a cubic activation function is shown in Figure 2. 


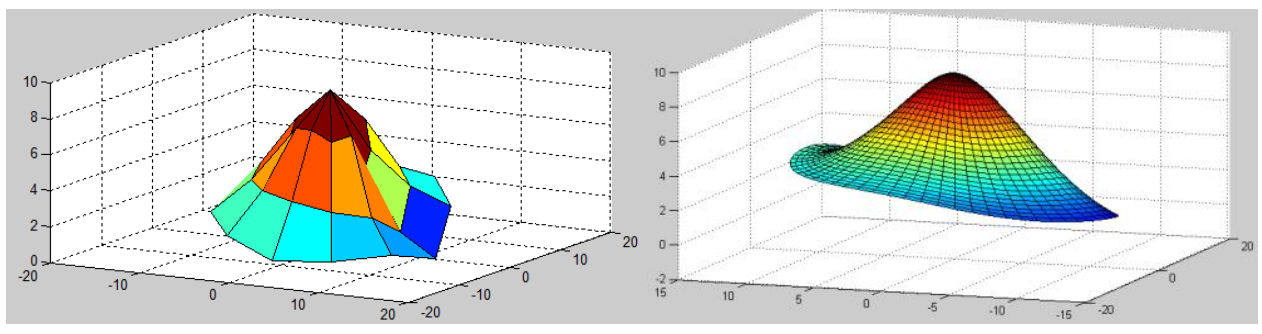

Fig. 2. A graphical representation of the obtained data can be seen on the left. The performance of the cubic activation function in the neural network is presented on the right.

The learning time of the neural network is tenths of a second. This is sufficient when using this method for tank batteries.

In this paper, the authors investigate the surface in a static state (effects of supplying the mixture to or pumping it from the tank in dynamics were neglected). They focus on measuring the volume of the "heap" formed by the material (the volume of the mixture under the heap remains unchanged), while estimating level values from its lower boundary. The volume of the mixture under the "heap", VocH, is calculated by the formula for the vertical cylinder volume.

The measurement of the volume by smooth surface was based on the average level of material with a smooth surface.

For the conical surface, the average angle of repose was equal to thirty-nine degrees due to unevenness and lumpiness, which is close to the tabulated values, and the angle of repose is chosen to be equal to forty degrees. When measuring the volume by the nonlinear function, the double integral was calculated by the Gauss-Kronrod method. The estimation of the errors for different volume measurement methods is presented in Table 2.

Table 2. Errors of methods.

\begin{tabular}{|c|c|c|}
\hline Smooth surface & Cone & $\begin{array}{c}\text { Nonlinear } \\
\text { function }\end{array}$ \\
\hline $25.8 \%$ & $21.7 \%$ & $8 \%$ \\
\hline
\end{tabular}

\section{Conclusion}

According to the experimental results, the method of volume measurement by smooth surface is found to have the greatest error. The error produced by the measurement method by cone is slightly less. By analyzing characteristics of materials in a more detail and developing the appropriate formulas, it is possible to increase the accuracy of measurement by cone. However, its main weaknesses will still exist - a narrow focus on the type of material and labour-intensive research.

Among three available methods, the method with approximation of a nonlinear function is the best one, since it allows the error to be controlled by the number of partitions of function responses or the number of experiments. Moreover, this method takes the surface model into account.

It is reasonable to use a RBF neural network for obtaining the surface model of granular substances in order to improve the accuracy of measuring volumes in tank batteries and storehouses of production enterprises.

The most effective techniques are those that are aimed at modeling surface functions and measuring their volume by quadrature or Monte Carlo methods, since these techniques, according to the obtained results, have the smallest error. In this case, the Gauss-Kronrod 
method is preferable, since its accuracy remains several times greater even if the difference in the number of operations is thousands of times.

The proposed method of volume measurement that takes a nonlinear surface function into account can also be applied to any other material. The measurement error will remain the same regardless of moisture content, flow and other rheological properties of materials.

\section{References}

1. T.S. Namazbaev, S.H. Esenbaev, V.A. Kolesnikov, Sredstva izmerenija i kontrolja tehnologicheskih processov na predprijatijah gorno-metallurgicheskogo kompleksa [Measurement and control tools of technological processes at the enterprises of the mining and metallurgical complex] (2012) (in Russian)

2. E.A. Yakimovich, N.V. Zamyatin, Information technologies in territorial management, industry, education, 165 (2002) (in Russian)

3. G.V. Smirnov, D.G. Smirnov, Proceedings of TUSUR University, 19(2), 99 (2016) (in Russian)

4. V.I. Makovkin, N.V. Zamyatin, Proceedings of TUSUR University, 19(4), 95 (2016) (in Russian)

5. Y. Huang, B. Chen, E. Pienkowski, G. Chen, H. Xiao. Sensor and Accurator A: Physical (2011)

6. S. Khaliq, S.W. James, R.P. Tatam, Opt. Lett., 26, 1224 (2001)

7. A.V. Kulazhskiy, Vestnik RGUPS, 95 (2009) (in Russian)

8. N.E. Konovalov, N.N. Sonne, I.G. Melnik, A.K. Yakimovich, Proceedings of GIPRODORNII, 18 (1976) (in Russian)

9. A.G. Rybakov, E.V. Lukyanova, I.S. Burmistrova, Yu.F. Muratova, M.V. Fyodorova, Journal "Power and Electrotechnical Systems", 430 (2015) (in Russian)

10. M.Ya. Vygodskiy, Spravochnik po vysshej matematike [Handbook of Higher Mathematics], 622 (AST, Moscow, 2006) (in Russian)

11. T.V. Filatova, Tomsk State University Journal, 284 (2004) (in Russian)

12. A.N. Kolmogorov, Doklady AN SSSR [Proceedings of the USSR Academy of Sciencies], 108, 179 (1956) (in Russian) 